\title{
Evaluation of ISI-Free Parametric Linear Combination Pulses in Digital Communication Systems
}

\author{
Cesar A. Azurdia-Meza ${ }^{1}$
}

Published online: 16 May 2015

(C) Springer Science+Business Media New York 2015

\begin{abstract}
Digital communication systems are characterized by having a filter at the transmitter and receiver sides to band limit the transmitted signals and diminish the effects to intersymbol interference (ISI). The optimum parametric linear combination pulse is evaluated at the transmitter and receiver sides by using different evaluation tools. The proposed pulse is evaluated in terms of the bit error rate in the presence of symbol-timing errors. Eye diagrams are presented to visually evaluate the susceptibility of the transmission system to ISI, and the maximum distortion is determined as a numerical measure of performance.
\end{abstract}

Keywords Bit error rate (BER) - Intersymbol interference (ISI) · Nyquist first criterion · Parametric linear combination pulse (PLCP)

\section{Introduction}

The rapid growth of digital communication systems over the last years requires higher error-free data rates and better bandwidth reuse. Current practical digital systems are band limited; hence, system designers have to transmit higher error-free data rates within a fixed limited bandwidth. Digital communication systems, with bandlimited channels, are characterized by having a filter at the transmitter and receiver side [1]. At the transmitter side, the data symbols, characterized as voltage levels or as impulses, are modulated and then filtered to fulfill a certain bandwidth constraint. At the receiver side, we need to design a filter that compensates for the distortion caused by the transmitter and the channel [1].

In a band limited digital communication system, channel distortion causes intersymbol interference (ISI). The design of intersymbol interference (ISI) free band limited signals

Cesar A. Azurdia-Meza

cazurdia@ing.uchile.cl

1 Department of Electrical Engineering, University of Chile, Santiago, Chile 
was a problem considered by Nyquist [1-3]. Nyquist's first criterion guarantees that a sequence of pulses, sampled at the optimum and uniformly spaced sampling instants, will be ISI-free. Besides the ISI-free prerequisite, pulse shaping filters have to exhibit low sensitivity to timing errors. In practical receivers, the presence of timing jitter causes the actual sampling points to deviate from the optimal ones; hence, symbol timing errors are produced. The prior implies that the tails of the filter must decay fast outside the pulse interval in order to eliminate the undesired effects of jitter.

To meet the prior constraints, several Nyquist pulses have been reported. The most popular ISI-free Nyquist pulse for distortionless transmissions is the raised cosine (RC) pulse [2-5]. Further, the RC pulse has been proposed by the 3rd Generation Partnership Project (3GPP) as the filter to be implemented at the user equipment (UE) and at the base station (BS), for transmission and reception [4, 5]. The so called "better than" raised cosine filter (BTRC), proposed in [6], and later derived in [3], outperforms the RC pulse in terms of larger eye opening and smaller bit error rate (BER). In [7], a linear combination between the RC pulse and the parametric linear pulse (PLP) was proposed to obtain a new family of ISI-free Nyquist pulses. The family of pulses proposed in [7] is known as the linear combination pulse (LCP). The LCP pulse was numerically optimized to outperform the BTRC pulse in terms of a wider eye opening and a smaller BER [7]. Other superior ISIfree pulses have been formulated, but will not be considered in this paper because they do not have an explicit time-domain expression [8-11].

The main goal of this manuscript is to investigate the performance of the optimized PLCP, originally derived in [12] for PAPR reduction, in terms of BER. The BER will be evaluated in the presence of time sampling errors. Other tools will also be implemented to evaluate the optimized PLCP. The maximum distortion and the eye opening of the optimized PLCP will be evaluated, and will be compared with other existing Nyquist filters with known explicit-time domain expressions.

\section{Nyquist Parametric Linear Combination Pulses}

Nyquist's first criterion guarantees that a sequence of pulses sampled at the optimum sampling instants will be ISI-free [2,3]. Nyquist's first criterion is given as

$$
h(p T)=\left\{\begin{array}{l}
1, p=0 \\
0, p= \pm 1, \pm 2, \pm 3, \ldots
\end{array},\right.
$$

where $h(t)$ is the impulse response, and $T$ is the symbol period. Whereas in the frequency domain, the Fourier transform of (1) is given as follows

$$
\sum_{m=-\infty}^{m=\infty} H\left(f+\frac{m}{T}\right)=T
$$

where $H(f)$ is the Fourier transform of $h(t)$. The excess bandwidth of a Nyquist ISI-free pulse is determined by the roll-off factor, $0 \leq \alpha \leq 1$, and $T=1 / 2 B$ is the symbol repetition rate for a bandwidth $B>0$.

According to [13], the linear combination of two pulses that comply with Nyquist's first criterion ensures that the resulting pulse will be ISI-free. In [12], a linear combination between two ISI-free parametric linear pulses (PLP) was proposed. In general, the PLP pulses and the BTRC pulse can be derived by applying the general parametric family of 
pulses defined in [3; Eq. (5)], in which $n \geq 0$ is a parameter that defines pulses with different decay rates [3]. The impulse response of the derived PLCP is described as follows [12]

$$
h(t)_{P L C P}=\mu h(t)_{P L P_{n=1}}+(1-\mu) h(t)_{P L P_{n=2}},
$$

where $\mu$ is the constant that corresponds to the linear combination, and it is defined for all real numbers [12, 14]. The linear combination constant adds an additional degree of freedom to design a pulse less sensitive to timing errors. The explicit time-domain expression of the PLCP pulse is given as

$$
h(t)_{P L C P}=\frac{\sin (\pi \tau)}{\pi \tau} \times \frac{4(1-\mu) \sin ^{2}(\pi \alpha \tau / 2)+\pi \alpha \mu \tau \sin (\pi \alpha \tau)}{\pi^{2} \alpha^{2} \tau^{2}},
$$

where $\tau$ is the normalized time $(\tau=\mathrm{t} / \mathrm{T}$ ), and $\alpha$ is the roll-off factor. The pulse defined in (4), evaluated for $t=0$, and for any value of $\mu$, is equal to one. Additionally, the PLCP, evaluated for $p= \pm 1, \pm 2, \pm 3, \ldots$, and for any value of $\mu$, is always equal to zero. Therefore, the proposed family of pulses in (4) fulfils Nyquist's ISI-free criterion, previously described in (1).

In [12], the PLCP pulse was optimized for PAPR reduction for the single carrier frequency division multiple access (SC-FDMA) system. It was found that the optimum linear combination constant is equal to 1.60. Therefore, in this paper we will evaluate the proposed pulse with $\mu=1.60$, for different roll-off factors and sampling timing errors to further validate the implementation of the optimum PLCP in digital communication systems.

The impulse response of the optimum PLCP and the other pulses used for comparison are plotted in Fig. 1. In most of our simulations we used a roll-off factor of 0.35 , which is a

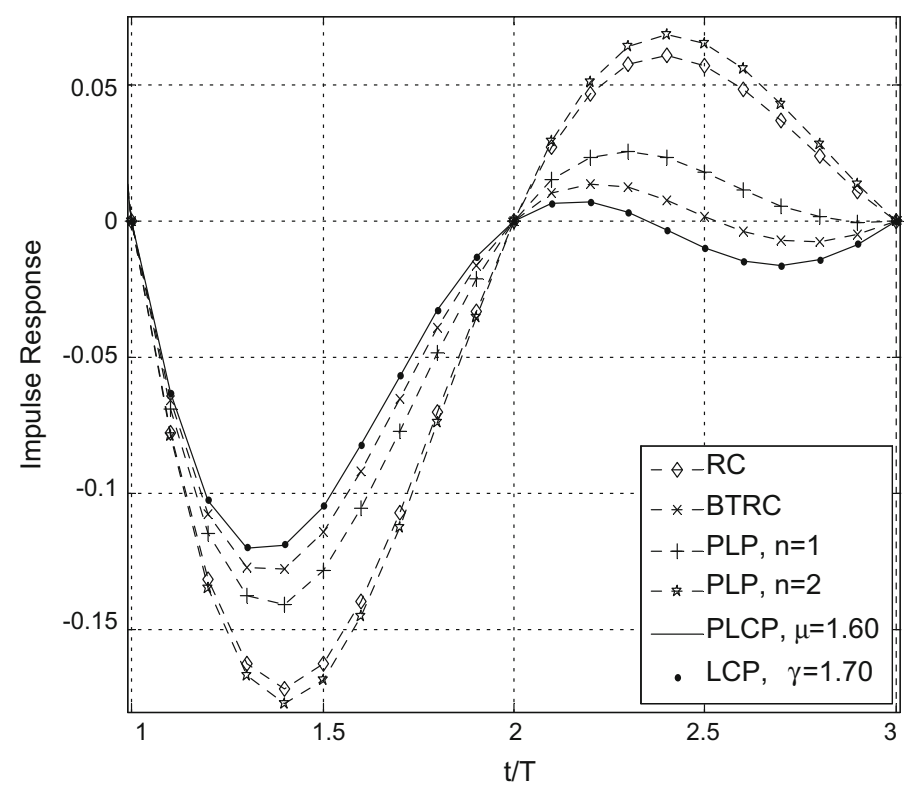

Fig. 1 Impulse response of the proposed pulse and other existing pulses with a roll-off factor $\alpha=0.35$ 
typical value used in literature [3, 6-13]. It can be seen that the ISI-free condition holds for the optimum PLCP. The proposed pulse has the smallest relative magnitudes in its two largest side lobes compared to those of the other evaluated pulses. The prior implies that the undesired effects of jitter will be diminished, and the optimum PLCP will be less sensitive to timing errors, resulting in a lower BER [3, 7-11]. Further, PAPR reduction can be achieved by minimizing the amplitudes of the two largest sidelobes $[12,15,16]$. It can be seen that the optimum PLCP has basically the same impulse response as the optimum LCP pulse. In [7], it was found that the optimum LCP pulse has a linear combination constant, $\gamma$, equal to 1.70 . Therefore, in this manuscript we will evaluate the LCP pulse with $\gamma=1.70$.

\section{Performance Evaluation}

In this section, we evaluate the performance of the proposed pulse by using several practical tools. The performance of the optimum PLCP will be compared with other Nyquist pulses with known explicit time-domain expressions.

One of the tools implemented to analyze the performance of the proposed pulse is the eye diagram, which is a mean of visually evaluating the vulnerability of the transmission system due to ISI $[1,10]$. We initially generated $10^{4}$ uniform random data points and implemented binary phase shift keying (BPSK) digital modulation. Next, we up sampled the transmitted sequence by inserting additional zeros. Later, we convolved the up sampled transmitted sequence with the pulses used for evaluation. Finally, we overlapped the time domain samples to plot the eye diagrams. The corresponding eye patterns are depicted in Figs. 2 and 3. For the sake of clarity and lack of space, only the outer and inner contour boundaries have been generated, corresponding to maximum and minimum distortion,

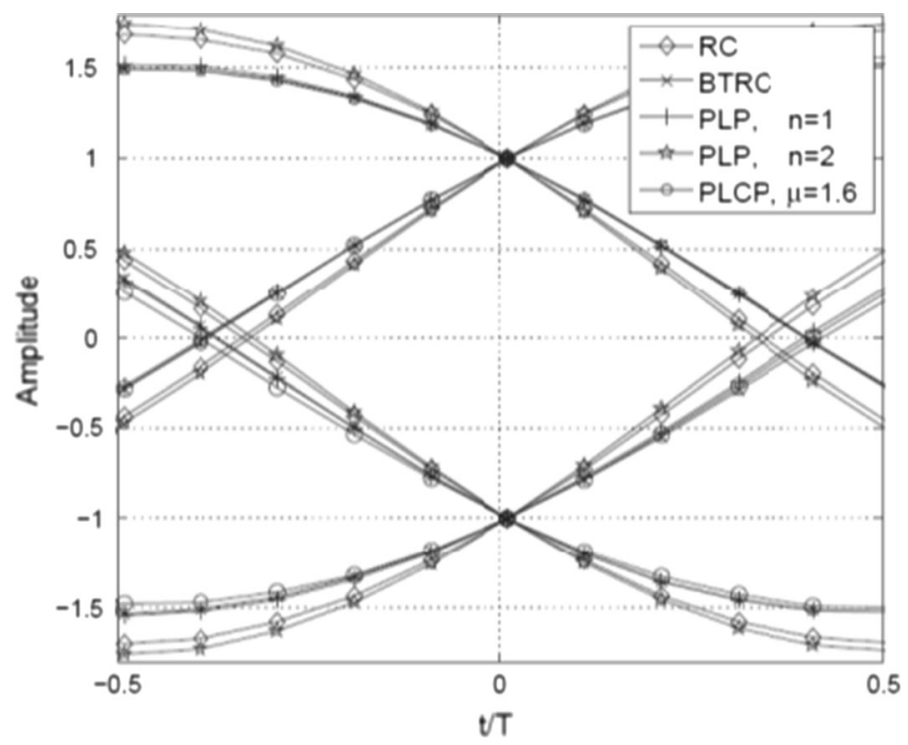

Fig. 2 Inner and outer contour eye diagram boundaries eye of the PLCP and other existing pulses with a roll-off factor $\alpha=0.35$ 


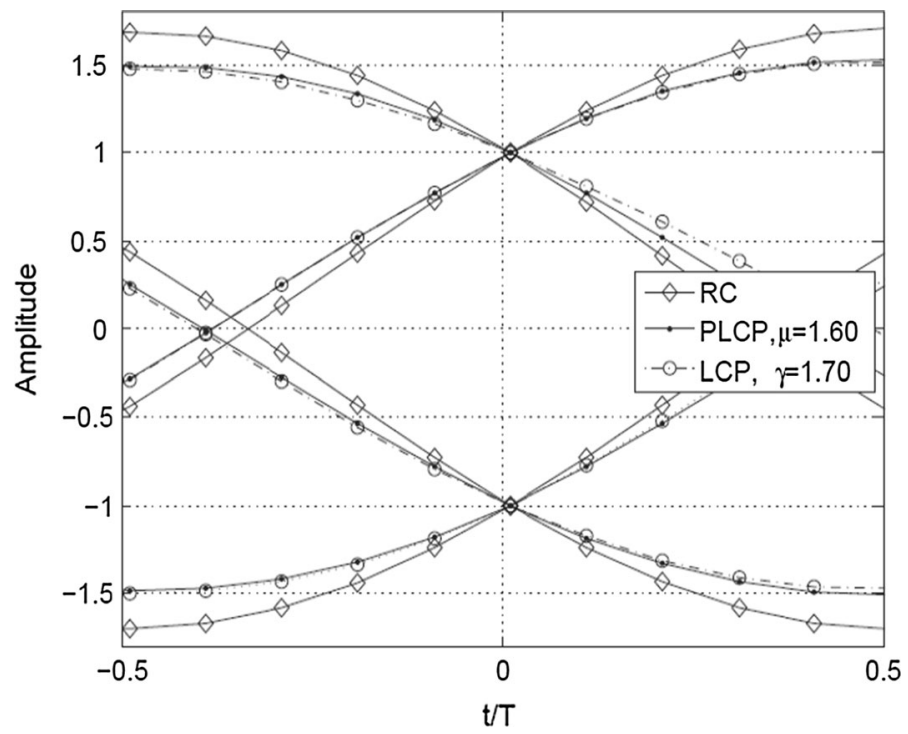

Fig. 3 Inner and outer contour eye diagram boundaries of the PLCP, LCP, and RC pulse with a roll-off factor $\alpha=0.35$

respectively. Observe in Fig. 2 that the BTRC and the proposed pulse exhibit a much wider eye opening than the parametric linear pulses (mainly PLP for $n=2$ ) and the RC pulse; hence, a lower BER is expected because these pulses deal better with ISI. This is despite the fact that the proposed optimum pulse and the BTRC pulse decay as $t^{-2}$, while the PLP pulse, for $n=2$, and the RC pulse decay as $t^{-3}$. Nevertheless, a lower decay rate is not always a disadvantage as long as the amplitudes of the two largest side lobes are diminished $[3,13,17]$. In Fig. 3 we plotted the outer and inner contour eye diagram boundaries of the optimum PLCP, LCP, and the traditional RC pulse. It can be seen that the eye diagram of the proposed PLCP pulse is almost identical as the one of the optimum LCP, and exhibits a much wider eye opening than the RC pulse.

The maximum distortion experienced by each pulse is a more quantitative measure of performance. It indicates the magnitude of the largest possible ISI sample at any given instant $[8,11]$. In all cases, the maximum distortion, which occurs at $t / T= \pm 0.5$, is smaller for the optimum PLCP and the optimum LCP, as depicted in Table 1. For comparison purposes, the maximum distortion was evaluated with different roll-off factors. It can be seen that the proposed optimum PLCP filter has the minimum maximum distortion for a

Table 1 Maximum distortion to ISI

\begin{tabular}{lll}
\hline Pulse & $\alpha=0.35$ & $\alpha=0.50$ \\
\hline RC & 1.6966 & 1.4786 \\
BTRC & 1.5065 & 1.3342 \\
PLP $(\mathrm{n}=1)$ & 1.5443 & 1.3545 \\
PLP $(\mathrm{n}=2)$ & 1.7470 & 1.5304 \\
LCP & 1.4778 & 1.3128 \\
PLCP & 1.4816 & 1.3108 \\
\hline
\end{tabular}


roll-off factor $\alpha=0.5$. For the case of a roll-off factor equal to 0.35, the optimum PLCP pulse has a similar minimum maximum distortion as the optimum LCP pulse, and outperforms the other pulses evaluated.

The last step of the evaluation process involves the calculation of BER in the presence of time sampling errors. The BER is the most important metric of performance because it considers the effects of synchronization, distortion, and noise. The error rates have been computed according to [17] for binary antipodal signals, and $2^{9}$ interfering symbols.

Let $\eta$ represent the jitter timing error. If the probability density function of the jitter timing error is defined as $\mathrm{f}(\eta)$, then the expected error probability due to ISI is defined as

$$
E\left[P_{e}\right]=\int P_{e}(\eta) f(\eta) d \eta
$$

where $P_{e}[\eta]$ is the error probability to ISI for a certain Nyquist pulse, and timing error $\eta$. Considering the case of additive white Gaussian noise (AWGN) in the channel, and binary antipodal signaling (BPSK), the error probability to ISI can be evaluated using the following truncated Fourier series [17]

$$
P_{e}(\eta)=\frac{1}{2}-\frac{2}{\pi} \cdot \sum_{\substack{m=1 \\ m=o d d}}^{M}\left\{\frac{\exp \left(-m^{2} w^{2} / 2\right) \sin \left(m w g_{0}\right)}{m}\right\} \prod_{\substack{k=N_{1} \\ k \neq 0}}^{N_{2}} \cos \left(m w g_{k}\right) .
$$

In (6), $M$ represents the number of coefficients considered in the approximate Fourier series, $w=2 \pi / T f$ where $T_{f}$ is the period used in the series, and $N_{1}$ and $N_{2}$ indicate the number of interfering symbols before and after the transmitted symbol. Whereas $g_{k}=$ $p(k T+\eta), p(t)$ is the Nyquist pulse implemented, and $T$ is the symbol period. A system signal-to-noise ratio (SNR) of $15 \mathrm{~dB}$ has been assumed, while different roll-off factors and sampling time errors were implemented to evaluate the performance of the optimum PLCP. The parameters used to evaluate the expression given in (6) for different Nyquist pulses are given in Table 2, and comply with the ones implemented in [7, 13, 17].

The obtained results are tabulated in Table 3. In general, it can be seen that by increasing the time sampling errors, the BER also increases. This is because when the received signal is sampled off center, ISI is produced and the BER increases [3]. The optimum PLCP yields smaller BERs compared to those of the other evaluated pulses, for different roll-off factors and timing jitter. However, the optimum PLCP pulse doesn't always outperform the optimum LCP pulse in terms of BER. A reason for this could be that

Table 2 Simulation parameters

\begin{tabular}{ll}
\hline Parameter & Value \\
\hline $\mathrm{M}$ & 31 \\
$\mathrm{~T}_{\mathrm{f}}$ & 30 \\
Interfering Symbols & 29 \\
$\mathrm{SNR}$ & $15 \mathrm{~dB}$ \\
Channel & $\mathrm{AWGN}$ \\
Modulation & $\mathrm{BPSK}$ \\
Roll-off factor & $\alpha=0.35,0.50$ \\
Symbol timing errors & $t / T= \pm 0.05, \pm 0.1, \pm 0.2, \pm 0.25$
\end{tabular}


Table 3 BER for $2^{9}$ interfering symbols, $\mathrm{SNR}=15 \mathrm{~dB}$, and different time sampling errors

\begin{tabular}{|c|c|c|c|c|c|}
\hline$\alpha$ & Pulse & $t / T= \pm 0.05$ & $t / T= \pm 0.1$ & $t / T= \pm 0.2$ & $t / T= \pm 0.25$ \\
\hline \multirow[t]{6}{*}{0.35} & $\mathrm{RC}$ & $5.9975 \mathrm{e}-8$ & $1.3894 \mathrm{e}-6$ & $9.7465 \mathrm{e}-4$ & $3.1987 \mathrm{e}-3$ \\
\hline & BTRC & $3.9355 \mathrm{e}-8$ & $5.4021 \mathrm{e}-7$ & $1.0132 \mathrm{e}-4$ & $9.3498 \mathrm{e}-4$ \\
\hline & PLP $\mathrm{n}=1$ & $4.3131 \mathrm{e}-8$ & $6.6009 \mathrm{e}-7$ & $1.3458 \mathrm{e}-4$ & $1.2251 \mathrm{e}-3$ \\
\hline & PLP $\mathrm{n}=2$ & $6.0027 \mathrm{e}-8$ & $1.2897 \mathrm{e}-6$ & $9.8762 \mathrm{e}-4$ & $3.2548 \mathrm{e}-3$ \\
\hline & $\mathrm{LCP}$ & $3.9176 \mathrm{e}-8$ & $5.0896 \mathrm{e}-7$ & $9.8871 \mathrm{e}-5$ & $9.2761 \mathrm{e}-4$ \\
\hline & PLCP & $3.9271 \mathrm{e}-8$ & $5.3872 \mathrm{e}-7$ & $9.9346 \mathrm{e}-5$ & $9.3184 \mathrm{e}-4$ \\
\hline \multirow[t]{6}{*}{0.50} & $\mathrm{RC}$ & $3.9723 \mathrm{e}-6$ & $5.4892 \mathrm{e}-7$ & $1.0223 e-5$ & $9.4691 \mathrm{e}-4$ \\
\hline & BTC & $2.4131 \mathrm{e}-8$ & $1.8578 \mathrm{e}-7$ & $2.0874 \mathrm{e}-6$ & $2.0151 \mathrm{e}-4$ \\
\hline & PLP n $=1$ & $2.6803 \mathrm{e}-8$ & $2.2957 \mathrm{e}-7$ & $2.7270 \mathrm{e}-6$ & $2.6163 \mathrm{e}-4$ \\
\hline & PLP $\mathrm{n}=2$ & $4.1083 e-6$ & $5.3768 \mathrm{e}-7$ & $1.0532 \mathrm{e}-5$ & $9.5174 \mathrm{e}-4$ \\
\hline & $\mathrm{LCP}$ & $2.3783 \mathrm{e}-8$ & $1.8469 \mathrm{e}-7$ & $1.9857 \mathrm{e}-6$ & $1.9842 \mathrm{e}-4$ \\
\hline & PLCP & $2.3856 \mathrm{e}-8$ & $1.8359 \mathrm{e}-7$ & $1.9743 \mathrm{e}-6$ & $1.9937 \mathrm{e}-4$ \\
\hline
\end{tabular}

the optimum PLCP has been optimized for different performance evaluations, as well as for PAPR reduction [12].

Overall, the proposed pulse exhibits an improved performance in terms of BER and maximum distortion. Moreover, the optimum PLCP outperforms the BTRC and LCP pulse, which are the best-known pulses with an explicit time-domain expression. A feasible option for implementing the proposed optimum PLCP would be to implement it as a finite impulse response (FIR) digital filter, as described in [18]. Consequently, the implementation of the proposed pulse is a viable option for digital communication systems with band limited channels.

\section{Conclusion}

In this manuscript, we evaluated the performance of the optimum PLCP at the transmitter and receiver side from different practical perspectives of interest. The proposed pulse exhibits the largest eye opening, and possesses the minimum maximum distortion compared to those of the other evaluated Nyquist filters with known explicit-time domain expressions. The optimum PLCP produces a smaller BER compared to those of the the other evaluated pulses, for different roll-off factors and timing offsets. Overall, the optimum PLCP outperforms the RC pulse, which is the pulse proposed by the 3GPP group. Further, the optimum PLCP and LCP pulses perform similarly.

Acknowledgments The authors acknowledge the financial support of the Program U-INICIA VID 2014, Grant No. UI-02/2014, University of Chile.

\section{References}

1. Sklar, B. (2001). Digital communications. New Jersey: Prentice-Hall.

2. Nyquist, H. (1928). Certain topics in telegraph transmission theory. AIEE Transactions, 47(2), 617-644.

3. Beaulieu, N. C., \& Damen, M. O. (2004). Parametric construction of Nyquist-I pulses. IEEE Transactions on Communications, 52(12), 2134-2142. 
4. 3rd Generation Partnership Project (3GPP) (2006). User equipment (UE) radio transmission and reception. Technical Specification Group Radio Access Network, Technical Report 25.101.

5. 3rd Generation Partnership Project (3GPP) (2009). Base station (BS) radio transmission and reception $(F D D)$. Technical Specification Group Radio Access Network, Technical Report 25.104.

6. Beaulieu, N. C., Tan, C. C., \& Damen, M. O. (2001). A "better than" Nyquist pulse. IEEE Communications Letters, 5(9), 367-368.

7. Azurdia-Meza, C., Lee, K. J., \& Lee, K. S. (2013). ISI-free linear combination pulses with better performance. IEICE Transactions on Communications, E96-B(2), 635-638.

8. Assimonis, S. D., Matthaiou, M., Karagiannidis, G. K., \& Nossek, J. A. (2011). Improved parametric families of intersymbol interference-free Nyquist pulses using inner and outer functions. IET Signal Processing, 5(2), 157-163.

9. Assalini, A., \& Tonello, A. M. (2004). Improved Nyquist pulses. IEEE Communications Letters, 8(2), 87-89.

10. Assimonis, S. D., Matthaiou, M., \& Karagiannidis, G. K. (2008). Two-parameter Nyquist pulses with better performance. IEEE Communications Letters, 12(11), 807-809.

11. Assimonis, S. D., Matthaiou, M., Karagiannidis, G. K., \& Nossek, J. A. (2009). Parametric construction of improved Nyquist filters based on inner and outer functions. In Proceedings international conference on communications, 1(1), 1-5.

12. Azurdia-Meza, C. A., Lee, K. J., \& Lee, K. S. (2012). PAPR reduction in SC-FDMA by pulse shaping using parametric linear combination pulses. IEEE Communications Letters, 12(12), 2008-2011.

13. Sandeep, P., Chandan, S., \& Chaturvedi, A. K. (2005). ISI-Free pulses with reduced sensitivity to timing errors. IEEE Communications Letters, 9(4), 292-294.

14. Strang, G. (1998). Introduction to linear algebra. Wellesley: Wellesley-Cambridge Press.

15. Azurdia-Meza, C. A., Lee, K. J., \& Lee, K. S. (2013). PAPR reduction in single carrier FDMA uplink by pulse shaping using a $\beta$ - $\alpha$ filter. Wireless Personal Communications, 71(1), 23-44.

16. Azurdia-Meza, C. A., Lee, K. J., \& Lee, K. S. (2012). PAPR reduction by pulse shaping using Nyquist linear combination pulses. IEICE Electronics Express, 9(19), 1534-1541.

17. Beaulieu, N. C. (1991). The evaluation of error probabilities for intersymbol and cochannel interference. IEEE Transaction on Communications, 39(12), 1740-1749.

18. Yao, C. Y. (2010). A design method of hybrid analog/asymmetrical-FIR pulse shaping filters with an eye-opening control option against receiver timing jitter. ETRI Journal, 32(6), 911-920.

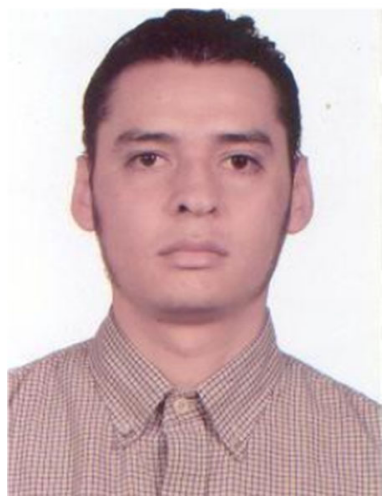

Cesar A. Azurdia-Meza received the B.Sc. degree in electrical engineering from Universidad del Valle de Guatemala, Guatemala in 2005, and the M.Sc. degree in electrical engineering from Linnaeus University, Sweden in 2009. In 2013 he obtained the Ph.D. degree in Electronics and Radio Engineering, Kyung Hee University, Republic of Korea. During his time as a Ph.D. candidate, he was a research assistant at the Advanced Wireless Communication Laboratory, Kyung Hee University. He joined the Department of Electrical Engineering, University of Chile as an Assistant Professor in August 2013. His research interests include OFDM, SC-FDMA, visible light communication systems, and signal processing for communication systems. 